\title{
The acidic activator GAL4-AH can stimulate polymerase II transcription by promoting assembly of a closed complex requiring TFIID and TFIIA
}

\author{
Weidong Wang, ${ }^{1,2}$ Jay D. Gralla, ${ }^{2}$ and Michael Carey ${ }^{3}$ \\ ${ }^{2}$ Department of Chemistry and Biochemistry and the Molecular Biology Institute, University of California, Los Angeles, Los \\ Angeles, California 90024-1569 USA; ${ }^{3}$ Department of Biological Chemistry, University of California at Los Angeles, School \\ of Medicine, Los Angeles, California 90024-1737 USA
}

The assembly of activated RNA polymerase II (pol II) transcription complexes has been investigated by assaying whether pre-assembly of intermediate complexes reduces the extended time required for start-site melting. The results show that a closed complex requiring factors IIA, IID, and the acidic activator GAL4-AH forms in a rate-limiting step. This directs the templates into a productive assembly pathway. Factor TFIIB is then added rapidly, affording further protection against diversion into nonproductive pathways. These events are followed by a series of rapid steps in which the remaining general factors are assembled onto the template, which is then melted using the energy of ATP hydrolysis.

[Key Words: Acidic activator; polymerase II; promoter melting; TFIIA]

Received April 21, 1992; revised version accepted June 23, 1992.

In both prokaryotes and eukaryotes, the pathway leading to transcription initiation proceeds sequentially through the steps of RNA polymerase promoter recognition, start-site melting, and production of transcript. The recognition step involves binding of polymerase to doublestranded DNA, either alone or in combination with accessory factors. The resulting assembly is referred to as the complete closed complex. This is followed by melting of the DNA around the start site to expose the template strand; this assembly is called the open complex. Subsequent addition of nucleoside triphosphates leads to initiation and elongation of transcription and a concomitant reclosing of the DNA around the start site. Transcriptional activators facilitate the assembly of these closed and open complexes by allowing them to form faster or more efficiently (for recent reviews, see Gralla 1990, 1991). The mechanism by which an activator elicits its effects is currently a major theme in gene regulation research.

In the absence of activator, the assembly of mammalian RNA polymerase II (pol II) transcription complexes on templates containing the TATA element is known to involve the sequential addition of transcription factors (TF) IID, IIA, IIB, pol II/IIF, and IIE (Buratowski et al. 1989; Flores et al. 1991; Greenblatt 1991; for review, see

${ }^{1}$ Present address: Howard Hughes Medical Institute, Beckman Center 209, Stanford University, Stanford, California 94305-5428 USA.
Roeder 1991; Zawel and Reinberg 1992). The resulting complex can initiate transcription rapidly in the presence of nucleoside triphosphates (Fire et al. 1984; Hawley and Roeder 1985) in a reaction that requires the energy of the $\beta-\gamma$ phosphoanhydride bond in ATP (or dATP) (Bunick et al. 1982; Sawadogo and Roeder 1984). The genes encoding several of these factors or their subunits have been cloned, including that for the TATA binding factor TBP (for review, see Greenblatt 1991), TFIIB (Ha et al. 1991), TFIIE (Ohkuma et al. 1991; Peterson et al. 1991; Sumimoto et al. 1991), and TFIIF (Sopta et al. 1989). TBP, is a subunit of TFIID that directly contacts the TATA box. TBP, in concert with the other general factors, is sufficient for basal-level, but not activated, transcription. A number of other subunits, called TBPassociated factors (TAFs) or coactivators, are required for activated transcription (Berger et al. 1990; Kelleher et al. 1990; Pugh and Tjian 1990, 1991; Dynlacht et al. 1991; Flanagan et al. 1991; Meisterernst and Roeder 1991; Meisterernst et al. 1991; Tanese et al. 1991).

Recently, we used potassium permanganate footprinting to show that in a HeLa cell nuclear extract the activator GAL4-VP16 was required for efficient melting of the transcription start site on DNA templates containing an adenovirus E4 promoter bearing upstream GAL4 binding sites (Wang et al. 1992). The opening or melting allows the polymerase access to the bases on the template and is thus a required event in gene activation. GAL4-VP16 was shown to function during closed com- 
plex assembly, at which time the general factors are added, but the precise activation step could not be identified. We now turn to a partially purified transcription system to define how an acidic activator stimulates assembly of pol II transcription complexes.

There have been several proposals addressing the steps at which activators intervene and at which point the DNA melts. DNA melting has been suggested to occur early in the pathway, either by the binding of TFIID (Mizutani et al. 1991), or after binding of both TFIID and TFIIB (Ha et al. 1991). Another study identified a copperphenanthroline-sensitive complex that required a complete complement of general factors to form (Buratowski et al. 1991). We proposed that these and other complexes generated in the absence of ATP are all closed complexes because start-site melting as measured by the permanganate assay has not yet occurred (Wang et al. 1992). Below, we confirm that melting is indeed a very late step in the initiation pathway.

The more critical problem is identifying at which point activator intervenes in the pathway. There have recently been several biochemical studies narrowing the steps at which this might occur (for review, see Lewin 1990; Ptashne and Gann 1990; Carey 1991; Sharp 1991). Recruitment of TFIIB into a transcription complex containing DNA-bound TFIID is stimulated by the acidic activator GAL4-AH, perhaps by direct binding to TFIIB (Lin et al. 1991). This was proposed to be the rate-limiting step in the assembly pathway (Lin and Green 1991). In other studies, the involvement of TFIID was inferred from its ability to contact acidic activators \{Stringer et al. 1990; Ingles et al. 1991) that lead to alterations in its interaction with promoter DNA in DNase I footprinting experiments (Horikoshi et al. 1988a,b). Indirect evidence led to the suggestion that activators may facilitate the entry of TFIIA into the preinitiation complex (Meisterernst and Roeder 1991; Meisterernst et al. 1991). Activators are also known to prevent transcriptional repression by nucleosomes (Taylor et al. 1991; Workman et al. 1991) and histone Hl (Croston et al. 1991; Laybourn and Kadonaga 1991). None of the processes described above requires nucleoside triphosphates, and thus activation in these cases appears to be a preinitiation event as opposed to the type of postinitiation activation that has been proposed for heat shock (Rougvie and Lis 1988) and other genes (for review, see Cullen 1990).

Below, we describe the use of direct kinetic studies and the permanganate open complex assay (SasseDwight and Gralla 1989) to determine the slow step in transcription complex assembly and the step at which activator intervenes to promote this assembly. Our results suggest that the activator promotes, either directly or indirectly, the function of TFIIA. Although this result was unexpected on the basis of the experiments of Lin and Green (1991), it is consistent with both that and the other aforementioned studies. Taken together with other experiments, the results imply that acidic activators and TFIIA assist in converting TFIID-containing complexes into productive closed complexes, thereby diverting them from less productive assembly pathways.

\section{Results}

The experimental system

The acidic activator used in this study is GAL4-AH (Lin et al. 1988; Carey et al. 1990a,b). The DNA template is a truncated adenovirus E4 promoter bearing its TATA sequence and 9 tandem repeats of the 17-bp synthetic binding site for the yeast activator GAL4 (Fig. IA). In a previous study using unfractionated HeLa extract, the acidic activator GAL4-AH stimulated transcription to the same level as GAL4-VP16 on this reporter template (Carey et al. 1990b). GAL4-AH is a stronger transcriptional activator than GAL4-VP16 in the factor reconstituted system at our standard low template concentration of $0.1 \mathrm{~nm}$ (possibly because GAL4-VP16 can squelch transcription more efficiently in that system; data not shown).

The general transcription factors and pol II were fractionated using modifications of standard procedures (Dignam et al. 1983; Reinberg and Roeder 1987; Reinberg et al. 1987). TFIID contained TBP and its associated TAFs. As shown below, one of our fractions contained trace amounts of pol II. We found that the HeLa cell TFIIB fraction could be replaced with recombinant protein in both transcription and open complex assays (Ha et al. 1991). Thus, the experimental system included a GAL4-responsive E4 template, various fractions containing partially pure HeLa transcription factors, and recombinant TFIIB and GAL4-AH, expressed in and purified from Escherichia coli. Transcription was measured by a primer extension assay.

Separate fractions containing TFIID, TFIIA, TFIIB, pol II, TFIIE/F, and GAL4-AH were all necessary for efficient transcription in a standard 1-hr transcription assay (Fig. 1B). The results, however, differed when our assay conditions corresponded to those used to assemble an open transcription complex (Fig. 1C). In this experiment, all of the components were incubated together for 30 min in the absence of ATP to allow assembly of the closed complex. Then all four nucleotides were added for 2 min to restrict transcription to a single round (Y. Jiang and J. Gralla, unpubl.). Significantly less RNA was produced, but more importantly, the system showed little dependence on the pol II fraction (Fig. 1C, cf. lane 6 to lane 21 . We believe polymerase is present at very low levels, perhaps in the TFIIE/F fraction (Reinberg and Roeder 1987). That amount, however, is apparently sufficient to transcribe the low concentration of template used in our first round transcription assay. The important point is that under conditions that were used to assay open complexes, transcription depended strongly on fractions containing all of the general factors and activator, but only weakly on added pol II.

\section{Factor requirements for pol II open complex formation}

$\mathrm{Next}$, we used the permanganate $\left(\mathrm{KMnO}_{4}\right)$ assay (SasseDwight and Gralla 1988, 1989; Zhang and Gralla 1989) to measure open complex formation in our reconstituted system. $\mathrm{KMnO}_{4}$ reacts selectively with single-stranded 
Figure 1. Factor dependence of in vitro transcription. $(A)$ Diagram of the truncated adenovirus E4 promoter, G9E4T (Carey et al. 1990 a,b). The multiple start-site region is marked with a bracket. $(B)$ Standard 1-hr transcription with a complete set of general transcription factors or with the indicated factors missing $(-1$. The arrow denotes the transcription signal. $(C)$ Same as $B$ except a one-round transcription assay was used (see text).
A

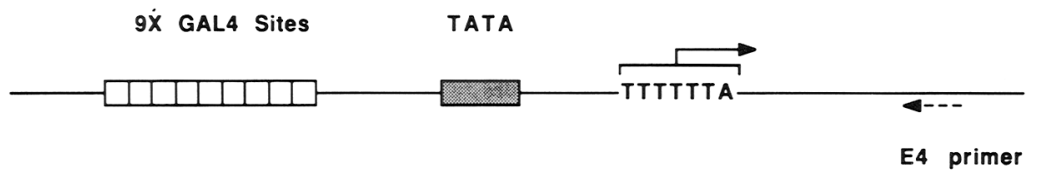

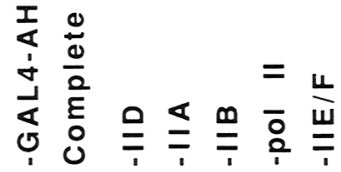
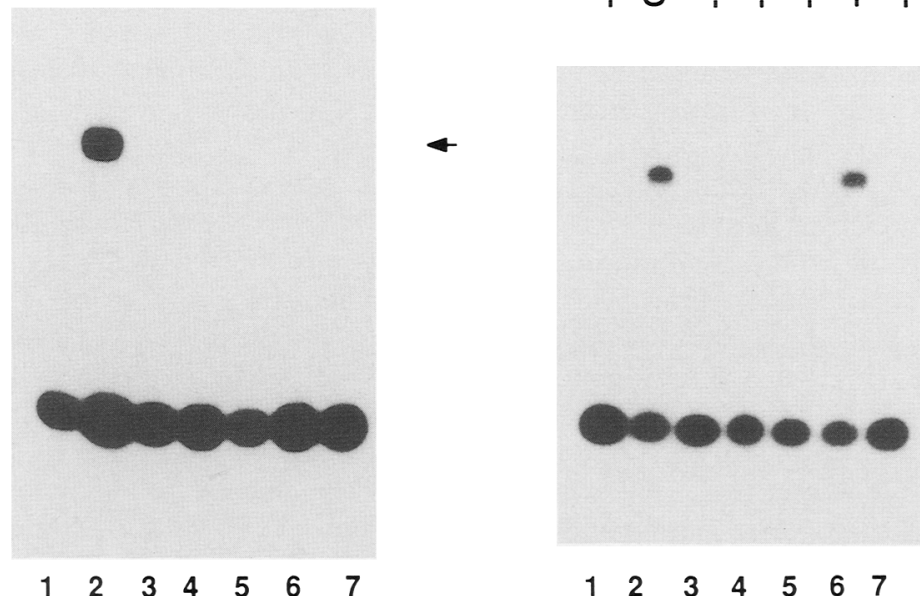

thymines in open complexes formed by pol II, pol III, bacterial RNA polymerases, and vaccinia initiation factors (Sasse-Dwight and Gralla 1988, 1989; Kassavetis et al. 1990; Vos et al. 1991; Wang et al. 1992). The E4 promoter contains 6 consecutive thymines in the initiation region that can act as start sites (Baker and Ziff 1981). When the initiation region is melted, these thymines become highly reactive to $\mathrm{KMnO}_{4}$. The modified residues serve as strong stops in primer extension assays using Taq DNA polymerase (Wang et al. 1992). We showed previously that these open complexes were functional. Addition of nucleoside triphosphates resulted in the disappearance of the hypersensitivity signal, consistent with polymerase elongating away from the initiation site and the DNA reclosing behind it. In contrast, addition of low concentrations of $\alpha$-amanitin prevented the disappearance, thereby implicating RNA pol II in the process (Wang et al. 1992).

The thymines in the start-site region of $\mathrm{E} 4$ promoter became hyperreactive to $\mathrm{KMnO}_{4}$ when a complete set of general factors and GAL4-AH were present (Fig. 2, lane $2)$. In the absence of GAL4-AH, however, these thymines reacted poorly with $\mathrm{KMnO}_{4}$ (lane 1), and their reactivities were similar to that of double-stranded DNA (Wang et al. 1992; also see Fig. 3, lane 1). This result indicates that in a reconstituted system, assembly of detectable pol II open complexes is completely dependent on the presence of activator as is the transcription observed under the same conditions (see Fig. 1B,C). We have shown previously that the relative level of open complex formation on templates bearing different numbers of GAL4 binding sites parallels the amount of transcription observed on these templates in our standard transcription assay (Carey et al. 1990b; Wang et al. 1992).

When either TFIID, TFIIA, TFIIB, or TFIIE/F were omitted from the complete reaction mixture, no open complex signal was observed (Fig. 2, lanes 3, 4, 5, and 7, respectively). Thus, we conclude that all the above general factors are required for open complex formation. As expected on the basis of the transcription results shown in Figure 1, open complexes can be seen in the absence of added pol II fraction (cf. lane 6 with lane 2), likely due to a low level of pol II contaminating the factors /detected in the sensitive single-round transcription assay of Fig. $1 \mathrm{Cl}$. Therefore, we cannot say for certain if melting requires pol II in addition to all the general factors.

\section{$\mathrm{KMnO}_{4}$ probing of intermediate complexes along the initiation pathway}

Next, we probed the intermediate complexes generated by sequential binding of TFIID, TFIIA, TFIIB, pol II, and TFIIE/F to the template (Fig. 3); GAL4-AH and dATP were present to promote closed complex formation and DNA melting, respectively. Potential intermediate complexes generated by TFIID, TFIIA, TFIIB, and pol II did not generate any open complex signal (lanes 3-6), indicating that only partially assembled closed complexes 


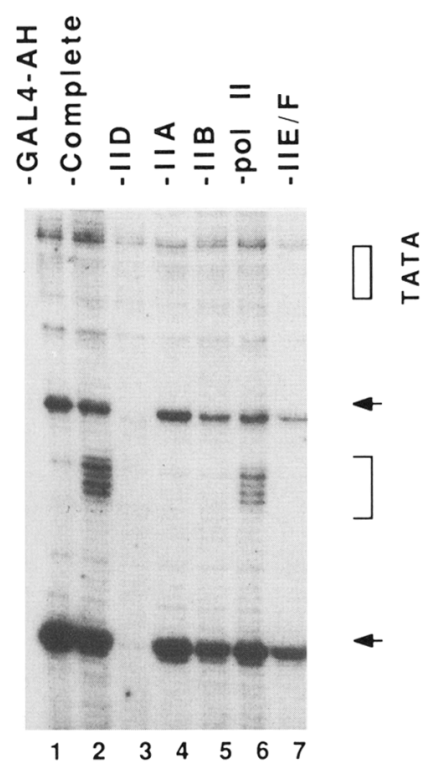

Figure 2. Requirements for open complex formation. The various reaction mixtures used in Fig. $1 \mathrm{C}$ were probed with $\mathrm{KMnO}_{4}$. The multiple start-site region is marked with a bracket. The arrows indicate the residues at -9 and +17 .

can form under these conditions. Addition of TFIIE/F was required to generate measurable open complexes. We conclude that none of the intermediate complexes expected to assemble under these various conditions are open complexes (lane 7).

Two hypersensitive bands at positions -9 and +17 can be seen in most of the lanes of Figure 3. These bands depend only on the presence of the TFIID fraction (Fig. 3) and can be observed when the template is incubated with the TFIID fraction alone (lane 3), even in the absence of activator (not shown, but see Fig. 2, lane 1). They do not appear when purified recombinant TFIID is used (Fig. 3, lane 2). Interestingly, neither band appears in naked DNA, and neither is completely dependent on addition of permanganate. These sites are not within the binding site of recombinant TBP, as measured by DNase I footprinting, but are within that of HeLa TFIID (Buratowski et al. 1988; Horikoshi et al. 1988a,b; Nakajima et al. 1988; Van Dyke et al. 1988). Thus, they appear to be influenced specifically by the binding of HeLa TFIID, which consists of TBP and its associated factors. These hypersensitive sites may be the result of DNA distortions caused by binding of the multicomponent TFIID complex to DNA surrounding the promoter start site. Chemically sensitive distorted DNA has been observed previously in bacterial repression complexes (Borowiec et al. 1987).

\section{Kinetic analysis of assembly of pol II open complex}

We showed previously that assembly of the pol II open complex at the E4 promoter in a HeLa extract proceeds through a slow, $\sim 20$-min activator-dependent, rate-lim- iting assembly of a closed complex, followed by a fast ATP-dependent step in which the DNA becomes melted; efficient closed complex formation depended on the activator GAL4-VP16 (Wang et al. 1992). Thus, both the rate-limiting step and the step facilitated by activator could in principle be any of the assembly steps. To identify the slow and activator-dependent steps in the pathway, we employed kinetic experiments.

In these experiments, we first determined the time required for complete open complex formation in the reconstituted system, beginning with free components. We then determined if preincubation of a subset of factors with the DNA template could reduce this time. Figure 4 shows the open complex signal at various times after initial mixing of all components. These autoradiographs were scanned by densitometry. Taking the level at $30 \mathrm{~min}$ as $100 \%$, the open complex was barely detectable at $5 \mathrm{~min}, 1 / 4$ complete at $10 \mathrm{~min}$, and about $3 / 4 \mathrm{com}$ plete at $20 \mathrm{~min}$. The quantitation of these and other experiments will be presented below; qualitatively, we used the appearance of the strong 20-min signal as a benchmark of substantial open complex formation. We note that the two bands that depend on the binding of TFIID were fully formed within $5 \mathrm{~min}$, when open com-

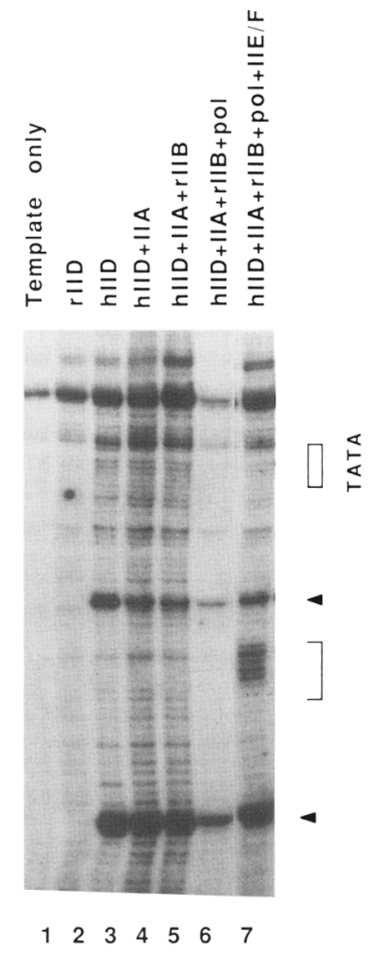

Figure 3. $\mathrm{KMnO}_{4}$ probing of intermediate complexes along the initiation pathway. The reaction mixtures contained the indicated general transcription components, and pol II, GAL4-AH, and dATP. After a short preincubation, the resulting complexes were probed using the permanganate assay. rTFIID represents the recombinant human TATA binding protein (TBP), whereas hTFIID represents the HeLa TFIID fraction containing TBP and TAFs. The multiple start-site region is marked with a bracket. The arrows indicate the residues at -9 and +17 . 


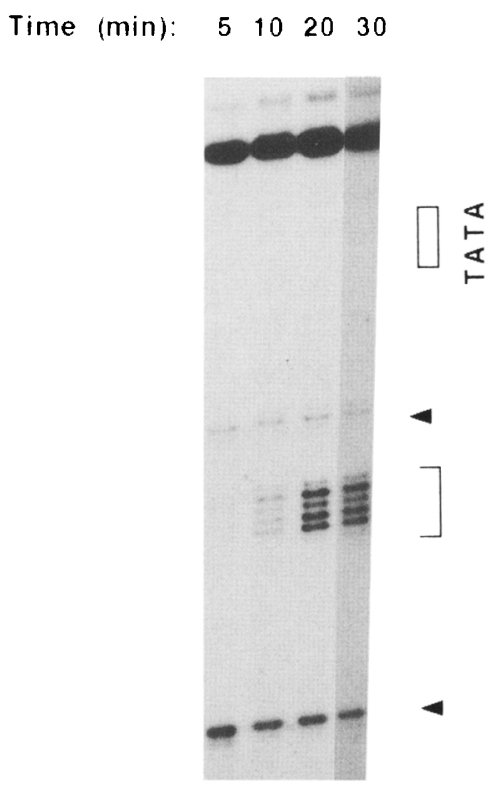

Figure 4. Kinetic studies of open complex assembly. Open complex formation was assayed at the times indicated after preincubation with a complete complement of factors and dATP. The multiple start-site region is marked with a bracket.

plexes are just beginning to form, demonstrating that this change occurred much more quickly than open complex formation. We used these bands to normalize the amount of open complex in the following experiments.

Next we determined whether preincubation of a particular subset of factors with DNA template could shorten the 20-min time period required to observe a strong open complex signal. During the assembly of complexes in the absence of activator, TFIID, TFIIA, and TFIIB assemble on the template first (Buratowski et al. 1989; Flores et al. 1991). Therefore, we preincubated various combinations of TFIIA, TFIID, and TFIIB in the presence and absence of activator. After $30 \mathrm{~min}$, these assembly reactions were chased with the remaining factors, dATP, and activator (if it was lacking in the preincubation). The goal was to determine if open complexes could now assemble rapidly during this chase; this would occur if the preincubation led to a complex that had already passed the slow rate-limiting step.

Figure 5A shows the results of an experiment in which TFIID (D), TFIID and TFIIA (DA), or TFIID, TFIIA, and TFIIB (DAB) were preincubated in the presence or absence of GAL4-AH for $30 \mathrm{~min}$. Each of these reactions was then chased for 5,10 , or 30 min prior to using the permanganate assay to measure open complexes. Recall from Figure 4 that in the absence of a preincubation, complexes are nearly undetectable at $5 \mathrm{~min}$. The results indicate that preincubation of DA or DAB in the presence of activator leads to significant open complex formation only $5 \mathrm{~min}$ after adding the remaining factors (Fig. 5A, lanes 7 and 13). In both cases, the levels of open complex observed after a 5-min chase were comparable to those seen if the chase was allowed to proceed for either 10 or $30 \mathrm{~min}$ (cf. lane 7 with lanes 8 and 9, or lane 13 with lanes 14 and 15). Figure 6 shows that a significant amount of open complex can even be observed after only a 2 -min chase reaction. In contrast, preincubation of the template with either TFIID (D) alone, or TFIID and TFIIB $\langle\mathrm{DB}\rangle$, did not result in the formation of open complexes after 5 min of chase (Fig. $5 A, B$ ). We infer that the TFIID and TFIIA are required to form a complex that can be rapidly converted to an open complex following addition of the remaining complement of factors and dATP.

The experiment in Figure 5 also shows that activator must be coincubated with TFIID and TFIIA for this rapidly converting complex to form. If activator is omitted from the preincubation but included in the chase, no significant open complex forms after 5 min even when preincubation includes DA or DAB (Fig. 5A, lanes 10 and 16). This is not due to slow binding by activator because DNase I footprinting shows that GAL4 derivatives bind completely within $2 \mathrm{~min}$ (not shown). We conclude that preincubation of either $\mathrm{DA}$ or $\mathrm{DAB}$ in the presence of activator has overcome the lengthy rate-limiting step required to assemble the open complex.

We note that preincubation mixtures lacking activator lead to a substantially decreased level of open complexes even after the extended 30-min chase period (Fig. 5). A similar result was observed if TFIIA was omitted from the preincubation. Therefore, it appears that in the absence of activator, the intermediate complexes are diverted into a nonproductive pathway. These cannot be efficiently rescued when activator is added subsequently. This phenomenon might be caused by the tight binding of negative regulatory factors to TFIID that interfere with subsequent assembly of productive complexes (Meinsterernst and Roeder 1991; Meisterernst et al. 1991).

These and other experiments were quantified by densitometry, and the amounts of open complexes were normalized, by comparing the intensity of the open complex signal to the +17 band that does not vary significantly in intensity and is completely formed at $2 \mathrm{~min}$ (Fig. 7). We estimate that the $t_{1 / 2}$ for the formation of open complexes from free components is $15 \mathrm{~min}$. In contrast, when the DA or DAB complexes are preformed and then chased, open complexes form with a $t_{1 / 2}$ of 2-3 min. Thus, the DA complex appears be have passed the ratelimiting step, because it can convert to the open complex five times faster than can free components.

Inclusion of TFIIB in the preincubation with TFIIA and TFIID increased the extent of open complex formation but did not measurably change the rate. Activator must be coincubated with DA and DAB for this increase in efficiency to occur. The lack of detectable rate enhancement was to be expected because TFIIB binds to a DA complex (Buratowski et al. 1989), and our experiments show that incubation of TFIID and TFIIA overcome the rate-limiting step. The result indicates that TFIIB plays an important role in protecting the DA complexes from becoming nonproductive, because coincubation with TFIIB leads to more open complexes. We conclude that TFIIB adds rapidly to protect the DA complexes. 

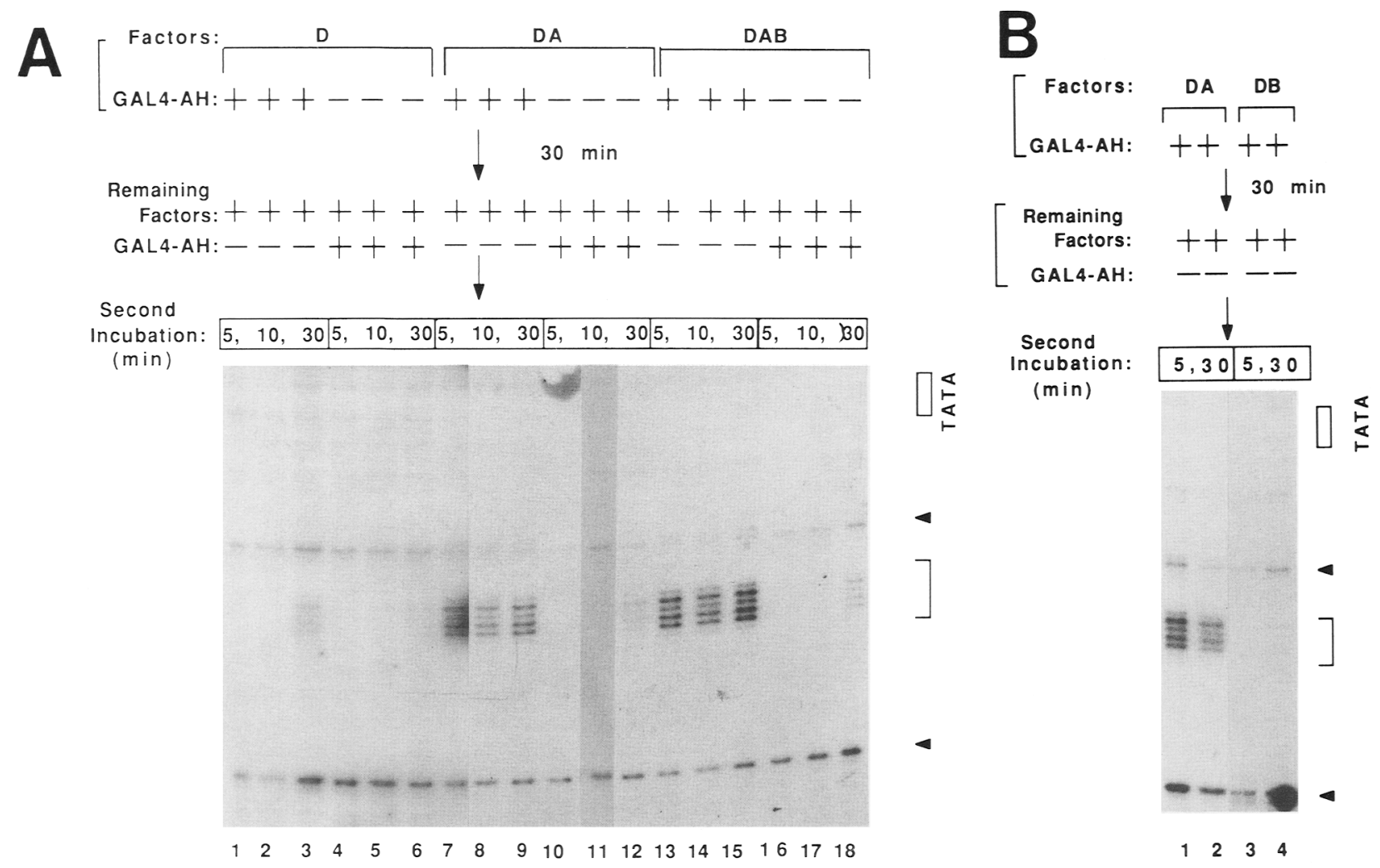

Figure 5. Kinetic analysis of polymerase II open complex assembly after preincubation of a subset of general factors $(A, B)$. The experimental procedure is diagrammed at the top of each lane. Abbreviations: (D) TFIID; (DA) TFIID and TFIIA; (DAB) TFIID, TFIIA, and TFIIB; (DB) TFIID and TFIIB. The presence $|+|$ or absence $(-\mid$ of factors and GAL4-AH are indicated. The multiple start-site region is marked with a bracket. All lanes in $A$ are from the same experiment except lane 11, which is from a separate experiment performed under identical conditions.

\section{Transcription assays suggest the same rate-limiting step}

The open complex kinetic experiments indicate that the rate-limiting step during assembly of a pol II open complex is the activator-dependent assembly of a closed complex requiring TFIID and TFIIA. To correlate these results with productive transcription initiation, we used a single-round transcription assay (as in Fig. 1B) to measure the rate at which transcriptionally competent complexes form. The protocol is identical to the open complex assay, except instead of adding dATP prior to $\mathrm{KMnO}_{4}$ probing, the four nucleoside triphosphates were added for 2 min before isolating RNA.

Lanes 1 and 2 of Figure 4 show the result obtained in the absence of preincubation. The transcription signal was weak $5 \mathrm{~min}$ after mixing and strong after $30 \mathrm{~min}$. This is consistent with results from the open complex assay shown in Figure 8, which demonstrated that few open complexes form at $5 \mathrm{~min}$, whereas many form by 30 min. Preincubation of DA or DAB with activator and template, however, allowed synthesis of a high level of RNA 5 min after the complementing factors were added (lanes 5 and 7), thus mimicking the open complex results. Preincubation in the absence of activator did not lead to formation of rapidly converting complexes (data not shown). The results show that preincubation mixtures including $\mathrm{DA}$ or $\mathrm{DAB}$ allowed closed intermediate complexes to form that could be rapidly converted into functional transcription complexes.

\section{Discussion}

Our results imply that the activator-dependent assembly of a complex requiring TFIID and TFIIA is the rate-limiting step in the assembly of transcriptionally active open promoter complexes (for summary, see Fig. 9). After fractions containing TFIID, TFIIA, and GAL4-AH are preincubated with template, the remaining factors and pol II can add to this preformed complex rapidly and, in the presence of ATP, melt the start-site with a half-time of $-3 \mathrm{~min}$ (Fig. 7). This is significantly shorter than the $t_{1 / 2}$ of 15 min observed for start-site melting beginning with free components. The comparison demonstrates that the rate-limiting step was bypassed by preincubation with TFIID and TFIIA. This rapid formation of open complexes from the preformed presumptive DA complex is paralleled by equally rapid formation of transcriptionally competent complexes (Fig. 8). Thus, out of the $\sim 15$ 
1

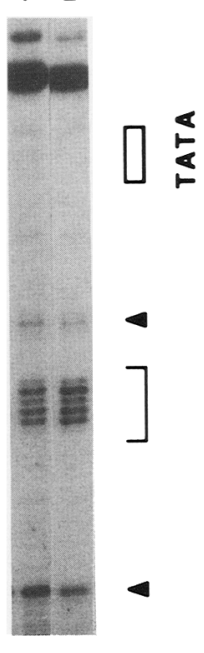

$\left[\begin{array}{cc}\text { Factors: } & \text { DA DAB } \\ \text { GAL4-AH: } & ++\end{array}\right.$

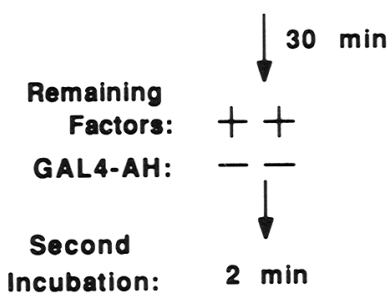

Figure 6. Preincubation of TFIID and TFIIA with GAL4-AH allows opening in just $2 \mathrm{~min}$. (See Fig. 5 legend for experimental procedures.)

min it takes to assemble a functional preinitiation complex in this system, $\sim 80 \%$ was consumed by forming a rate-limiting complex that required factors TFIID and TFIIA.

The assembly of the rapidly converting complex also required the activator GAL4-AH. If the activator was absent during the preassembly reaction including TFIID and TFIIA, then a complex that could not be rapidly opened formed, even when the activator was added subsequently. Thus, the activator assists during or immediately after recruitment of TFIIA into a preinitiation com. plex containing TFIID. This process could be facilitated by direct interaction of activator with either the TBP component (Stringer et al. 1990; Ingles et al. 1991) or the TAF component (Pugh and Tjian 1990; Dynlacht et al. 1991; Tanese et al. 1991) of TFIID, or through the structural changes in the TFIID complex caused by activator (Horikoshi et al. 1988a,b). The requirement for activator at this step, however, does not preclude its involvement at later steps in the assembly pathway, as discussed below.

In addition, the results of Figure 5 also suggest that the activator prevents formation of a complex that is refractory to the assembly of an open complex on addition of the missing components. When partial complexes are

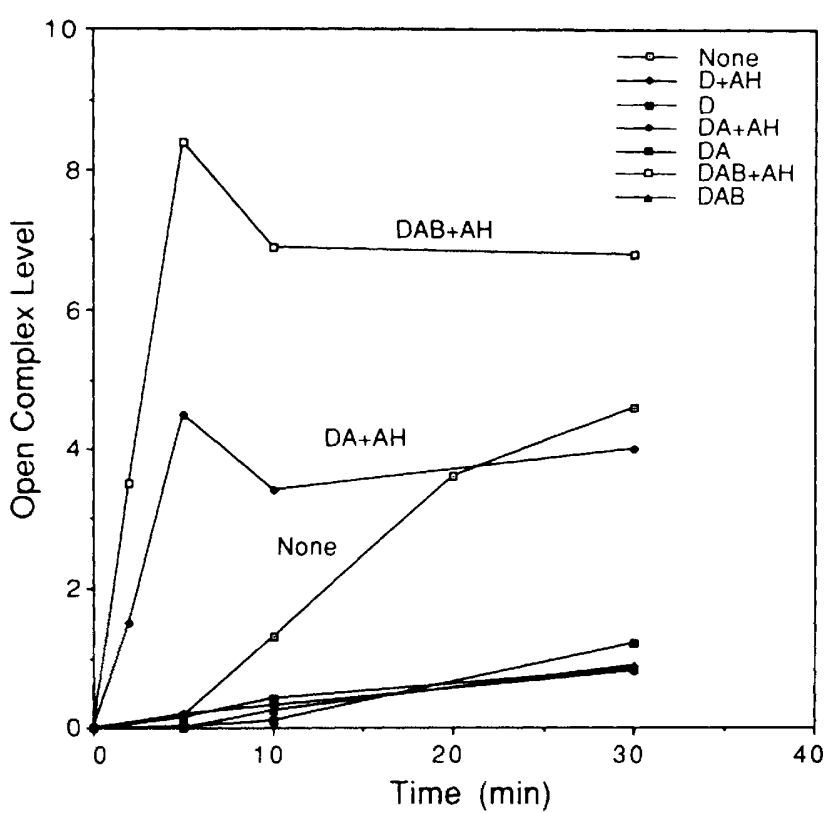

Figure 7. Rate of open complex formation starting from various intermediates. The data from several experiments were averaged. The abbreviations for $\mathrm{D}, \mathrm{DA}$, and $\mathrm{DAB}$ have been described in the Fig. 5 legend. Other abbreviations: $(A H)$ GAL4 $\mathrm{AH}_{i}$ (none) the kinetic data when no preincubation was performed.

assembled in the absence of activator and TFIIA, a full level of open complex formation cannot be attained, even when the missing factors are added subsequently. One likely cause of this is the binding of negative cofactors (Meisterernst and Roeder 1991; Meisterernst et al. 1991). These factors can compete with TFIIA to bind the

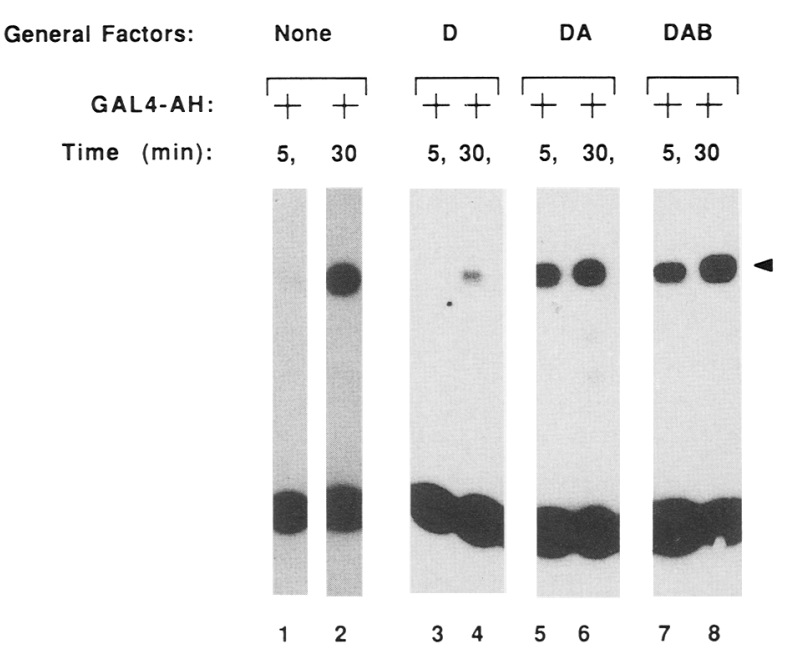

Figure 8. Transcription assay showing rapid starting from a preformed complex. The experimental procedures are the same as Fig. 5, except a one-round transcription was used instead of $\mathrm{KMnO}_{4}$ probing. The indicated fractions were preincubated with GAL4-AH, and then the remaining factors were added for the times indicated. 


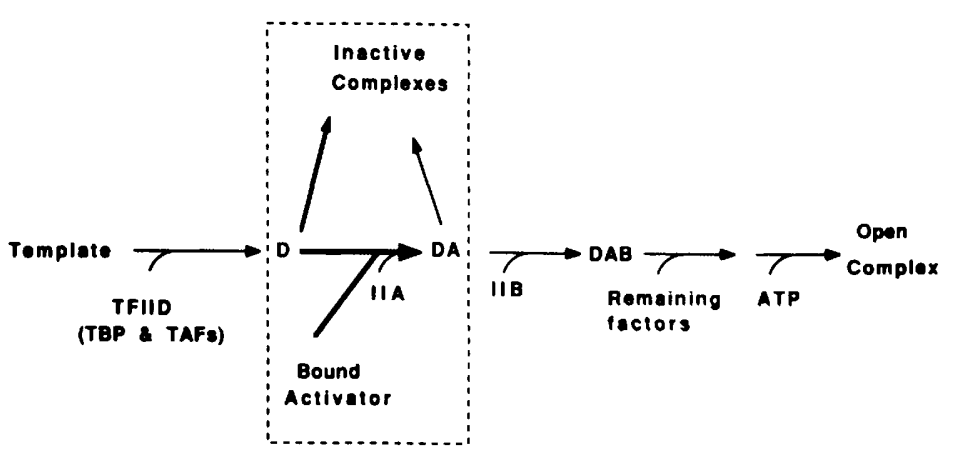

Figure 9. Summary of assembly of a polymerase II open complex on the GAL4-responsive E4 promoter.

TFIID complex (TBP + TAFs) and repress transcription. Thus the role of activator during the rate-limiting step could be indirect. That is, its binding might favor a conformation of a complex that binds TFIIA and TFIIB rather than negative cofactors.

The negative cofactors might represent TAFs for other nuclear polymerases. TBP is now known to be required for transcription by polymerases I, II, and III (Cormack and Struhl 1992; Schultz et al. 1992), and the associated TAFs in each case are thought to be distinct (Timmers and Sharp 1991; Comai et al. 1992). Binding of pol I or pol III TAFs to a pol II-responsive TFIID might lead to inhibition of transcription. The role of activator and TFIIA would then be to prevent this binding, thereby promoting formation of a pol II complex. In a related scenario, entire complexes of TBP and TAFs required for transcription by pol I and pol III might be contaminating our TFIID fraction. If all are able to bind a TATA box, then the role of activator and TFIIA would be to select the pol II-responsive form and recruit it to the promoter. It is important to emphasize, however, that a biological interpretation of the inhibition results must be regarded with caution because the use of impure fractions might tend to emphasize a process that does not occur in vivo.

The results (Fig. 7) show that addition of TFIIB also prevents diversion to repressed complexes. Thus, once TFIIA is bound, recruitment of TFIIB by activator (Lin et al. 1991) could further protect against competition by negative cofactors (Meisterernst and Roeder 1991; Meisterernst et al. 1991). The involvement of activator at more than one step could account for its ability to activate transcription synergistically (Meiklejohn and Gralla 1989; Carey et al. 1990a,b).

This model can be used to explain the reported variabilty in the requirement for factor TFIIA in reconstituted transcription systems (for review, see Roeder 1991). The systems may vary in the balance between TFIIA and negative cofactors. When the experimental system contains few negative cofactors, then the requirement for TFIIA would be much less because there would be no need to protect against diversion into nonproductive pathways. One study of a TFIIA-independent system (Lin and Green 1991) showed that the same activator used here, GAL4-AH, was required for the recruitment of TFIIB into the preinitiation complex. That result is consistent with this study in that recruitment of TFIIA can precede binding of TFIIB, and thus the activator could recruit them both. Alternatively, TFIIA-independent systems may contain TFIIA in the TFIID fraction (Meinsterernst and Roeder 1991) or TFIIG, which can partially substitute for TFIIA (Sumimoto et al. 1990). There have not yet been kinetic measurements on systems lacking added TFIIA.

Our results indicate that all of the general factors, including TFIID, TFIIA, TFIIB, and TFIIE/F, are required before the DNA can be melted, as speculated previously (Wang et al. 1992). We do not have conclusive evidence that pol II itself is required for open complex formation, due to the presence of very low levels of contaminating pol II in our system. However, because TFIIF joins the complex during pol II binding (Buratowski et al. 1989) and TFIIE afterwards (Flores et al. 1991), and because the TFIIF fraction is required for open complex formation, pol II should also be required. Because TFIIE binds after pol II/TFIIF (Flores et al. 1991), it is possible that TFIIE is not required to form the open complex. Future experiments will be required to resolve this issue and to determine the roles of the recently identified factors TFIIG (Sumimoto et al. 1991), TFIIH, and TFII) (for review, see Zawel and Reinberg 1992).

It is interesting to note that the activator enters the pathway very early, directly after the promoter is marked by the binding of the TFIID complex. This may be the earliest step that can provide the diversity necessary for appropriate gene regulation. It has been argued that the promoter should be marked by binding of at least one factor prior to activation to provide a target for the looping of upstream activators (Gralla 1991). In the step after the promoter is marked, there appears to be a competition between activator-mediated productive complex assembly and negative cofactors (Meisterernst and Roeder 1991; Meisterernst et al. 1991), which divert the complex. This early entry of activator may also allow the establishment of an intermediate transcription complex containing TFIIA that need not be disrupted when the polymerase leaves during transcription. Because assembly of an open complex from a TFID-TFIIA-activator complex is very rapid, this would allow subsequent 
rounds of assembly and transcription to be very rapid, as we have recently observed (Y. Jiang and J. Gralla, unpubl.). Only when activator is withdrawn would rapid transcription cease as the negative cofactors would begin to outcompete TFIIA. These speculations require further investigation into the pathway of open complex formation in the presence of other activators and negative cofactors.

\section{Materials and methods}

DNA templates

The plasmid G9E4T has been described previously /Carey et al. $1990 \mathrm{a}, \mathrm{b})$. It contains 9 GAL4 binding sites 23 bp upstream of the truncated adenovirus E4 promoter TATA box. The E4 primer was used previously for both transcription and open complex studies. Its $5^{\prime}$ end is located $\sim 90$ bp downstream of the start site and reads the top nontemplate strand of the DNA. The DNA sequence of the region encompassing the E4 start-site promoter $(-28$ to +18$)$ is as follows: 5'-TATATATAGACTCTCTGCACITGGCCCTTTTTTACACTGTGACTGATT- $3^{\prime}$. The underlined residues indicate the $T s$ in the start-site region that become melted in the initiation complex (Wang et al. 1992). The TATATATA sequence at the $5^{\prime}$ end represents the E4 TATA box.

\section{Preparation of transcription factors}

The general transcription components were purified by the methodology of Roeder (Dignam et al. 1983) with minor modifications. Briefly, $40 \mathrm{ml}(250 \mathrm{mg})$ of HeLa cell nuclear extract (Dignam et al. 1983) was applied to a 30-ml P-11 (Whatman) column pre-equilibrated in buffer $\mathrm{D}[20 \mathrm{mM}$ HEPES ( $\mathrm{pH} 7.9\}, 0.1$ mM EDTA, $1 \mathrm{mM} \mathrm{DTT}$, and $1 \mathrm{mM}$ PMSF] containing $0.1 \mathrm{M} \mathrm{KCl}$. The column was then washed with buffer $\mathrm{D}+0.1 \mathrm{M} \mathrm{KCl}$ and developed sequentially with steps of buffer $\mathrm{D}$ containing $0.3,0.5$ (TFIIB, TFIIE, TFIIF, pol II), and $0.85 \mathrm{M} \mathrm{KCl}$ (TFIID).

The P-11 flowthrough containing TFIIA was applied directly to a $10-\mathrm{ml} \mathrm{DE}-52$ column pre-equilibrated in buffer $\mathrm{D}$ containing $0.1 \mathrm{M} \mathrm{KCl}$. The column was then washed with $30 \mathrm{ml}$ of $\mathrm{D}+0.1 \mathrm{M} \mathrm{KCl}$ and TFIIA activity was eluted with buffer $\mathrm{D}+0.3 \mathrm{M} \mathrm{KCl}$.

The $0.5 \mathrm{M} \mathrm{P}-11$ fraction was dialyzed against buffer $\mathrm{D}+0.15$ $\mathrm{M} \mathrm{KCl}$ and applied to a $10-\mathrm{ml} \mathrm{DE}-52$ column pre-equilibrated in the same buffer. TFIIB activity flowed through the column, and pol II, TFIIE, and TFIIF (referred to as TFIIE/F in the text) were eluted with buffer $\mathrm{D}+0.3 \mathrm{M} \mathrm{KCl}$. The eluate was dialyzed down to $\mathrm{D}+0.15 \mathrm{M} \mathrm{KCl}$, reapplied to $\mathrm{DE}-52$, and eluted again with buffer $\mathrm{D}+0.3 \mathrm{M} \mathrm{KCl}$. This removed the residual TFIIB and much, but not all, pol II.

The $0.85 \mathrm{M}$ P-11 fraction containing TFIID activity was dialyzed against buffer $\mathrm{D}+0.05 \mathrm{M} \mathrm{KCl}$ and applied to an $8-\mathrm{ml}$ DE-52 (Whatman) column. After washing extensively with preequilibration buffer, TFIID was eluted with buffer $D$ containing $0.2 \mathrm{M} \mathrm{KCl}$. This eluate was applied directly to a 3-ml heparinSepharose (Pharmacia) column pre-equilibrated in buffer $\mathrm{D}+0.3 \mathrm{M} \mathrm{KCl}$. The column was washed with the pre-equilibration buffer and TFIID activity was eluted with buffer $\mathrm{D}+0.5 \mathrm{M} \mathrm{KCl}$.

RNA pol II was purified essentially as described (Reinberg and Roeder 1987). Pol II and the factors were dialyzed against $0.5 \times$ buffer $D+0.05 \mathrm{~m}$ for use in cell-free transcription experiments.

TFIIB was overexpressed in and purified from $E$. coli using an expression vector kindly provided by $\mathrm{D}$. Reinberg $(\mathrm{Ha}$ et al.
1991). Six liters of $E$. coli [BL21(DE3)] harboring the expression vector were grown to an $A_{600}$ of 0.5 , and TFIIB expression was induced by addition of IPTG to $1 \mathrm{~mm}$. After $3 \mathrm{hr}$ the cells were harvested, washed in 1 liter of buffer A 20 mM HEPES (pH 7.9) and $0.2 \mathrm{M} \mathrm{NaCl}$, and resuspended in buffer A containing $20 \mathrm{mM}$ 2-mercaptoethanol and $1 \mathrm{mM}$ PMSF. The cells were then lysed by sonication, and the insoluble debris was removed by centrifugation at $10,000 \mathrm{~g}$, for $10 \mathrm{~min}$. Polyethylenimine (PEI) was added dropwise to the supernatant while stirring gently, to a final concentration of $0.1 \%$. After $30 \mathrm{~min}$ at $0^{\circ} \mathrm{C}$, the precipitate was removed by centrifugation for $10 \mathrm{~min}$ at $10,000 \mathrm{~g}$. Ammonium sulfate (0.2 grams) was added per milliliter of supernatant. After $30 \mathrm{~min}$, the precipitate containing about $40 \%$ of the starting TFIIB was collected by centrifugation as above. The pellet was resuspended by Dounce homogenization in buffer $\mathrm{C}[20 \mathrm{mM}$ HEPES (pH 7.9), $1 \mathrm{mM}$ EDTA, $0.5 \mathrm{mM}$ PMSF, $10 \mu \mathrm{g} / \mathrm{ml}$ of leupeptin and pepstatin, $20 \mathrm{~mm} 2$-mercaptoethanol] to a conductivity equal to buffer $\mathrm{C}+0.2 \mathrm{M} \mathrm{NaCl}$. Insoluble debris was removed by centrifugation. The supernatant containing about $30 \%$ of the original TFIIB was applied to a $3-\mathrm{ml} \mathrm{P-11}$ column pre-equilibrated in buffer $\mathrm{C}+0.2 \mathrm{M} \mathrm{NaCl}$. The column was washed with buffer $C+0.2 \mathrm{M}$, and TFIIB activity was eluted with buffer $\mathrm{C}+0.6 \mathrm{M} \mathrm{NaCl}$.

GAL4-AH was purified as described previously (Lin et al. 1988).

\section{In vitro transcription reactions}

The 40- $\mu$ l mixture contained $10 \mathrm{ng}$ of supercoiled G9E4T template, $200 \mathrm{ng}$ of pGEM- 3 carrier DNA, $8 \mathrm{~mm} \mathrm{MgCl} 2,4 \mu \mathrm{g}$ of BSA, $15 \mu \mathrm{l}$ of TFIID, $10 \mu \mathrm{l}$ of TFIIA, $1 \mu \mathrm{l}$ of TFIIB, $1 \mu \mathrm{l}$ of pol II, and $3 \mu \mathrm{l}$ of TFIIE/F. For transcription assays, a mixture of ATP, UTP, GTP, and CTP was added to a final concentration of 0.5 $\mathrm{mM}$. For open complex assays, a mixture of dATP and $\alpha$-amanitin was added to a final concentration of $0.5 \mathrm{mM}$ and $1 \mu \mathrm{g} / \mathrm{ml}$, respectively. All reactions were performed for $1 \mathrm{hr}$ at $30^{\circ} \mathrm{C}$.

In these transcription assays, all 4 nucleoside triphosphates were added at the beginning of the incubation. Omitting TFIIA or pol II in the mixture resulted in slightly higher signals than background, but their levels were insignificant when compared to that of a complete set of factors. The basal transcription level is low, usually indistinguishable from the background; the template DNA concentration was $10 \mathrm{ng} / 40 \mu \mathrm{l}$. As a result, our system strongly responded to GAL4-AH for activation. Basal transcription was observed when higher DNA concentrations were used (data not shown).

The 1-hr transcription protocol described above measures RNA produced during multiple rounds of transcription because nucleotide substrates were present during the entire incubation. On the other hand, the $\mathrm{KMnO}_{4}$ assay is different from the transcription protocol in that no nucleotide substrates were added. Therefore, it only measures the open complex generated in the first round. To correlate the transcription protocol and the open complex assay, we revised the transcription protocol to allow the transcription complex to assemble for $30 \mathrm{~min}$, followed by a 2-min incubation with nucleotide substrates. The kinetic experiments showed that the open complex level began to plateau between 20 and $30 \mathrm{~min}$. Thus this protocol measures RNA produced largely from the first round.

In the absence of added pol II, the remaining factors gave a significant amount of transcripts in the one-round transcription assay but not in the multiple-round assay. We speculate that the trace amount of pol II might be inactivated by phosphorylation after the initial round of transcription (Laybourn and Dahmus 1990; Lu et al. 1991), and cannot be used for subsequent rounds. It is also possible that pol II was inactivated by nonspecific 
kinases using NTPs added during the lag period used to assemble preinitiation complexes.

We attempted to use recombinant TFIIE (kindly provided by M.G. Peterson; Peterson et al. 1991) and recombinant TFIIF (kindly provided by Z. Burton; Sopta et al. 1989) to replace our HeLa TFIIE/F fraction. The open complex signals, however, were too weak for us to detect.

\section{Potassium permanganate probing}

The potassium permanganate probing of open complexes on the E4 promoter has been described previously (Wang et al. 1992). The following conditions were used for the in vitro probing in this study. Instead of ATP, dATP was used in all experiments to provide the energy for DNA melting. This substitution was carried out because there appear to be endogenous nucleoside triphosphates that support elongation, and consequently result in a decrease in open complex signals: ATP, but not dATP, can be used as an elongation substrate. In our standard procedure, the template was first incubated with the transcription factors for $30 \mathrm{~min}$. Then $\alpha$-amanitin was added together with dATP for 2 min before $\mathrm{KMnO}_{4}$ probing. $\alpha$-Amanitin is a pol II elongation inhibitor. It is added to the incubation mixtures to prevent fortuitous escape of polymerase from the open complex in the presence of low concentrations of nucleoside triphosphates that may occasionally contaminate our less pure factor preparations. The contamination is more apparent in crude nuclear extracts (see Wang et al. 1992). Preincubation with $\alpha$-amanitin does not affect the rate at which the open complexes form. The final concentration of $\alpha$-amanitin and dATP are $1 \mu \mathrm{g} / \mathrm{ml}$ and $0.5 \mathrm{~mm}$, respectively. The $\mathrm{KMnO}_{4}$-modified template was subsequently purified and analyzed by primer extension as described (Wang et al. 1992).

\section{Kinetic studies using open complex assay}

In experiments performed without a preincubation, the template was incubated with all the general factors, including TFIIA through F, pol II, and GAL4-AH for 3, 8, 18, and $28 \mathrm{~min}$. Then, $\mathrm{dATP}$ and $\alpha$-amanitin were added for an additional $2 \mathrm{~min}$ before $\mathrm{KMnO}_{4}$ probing. Thus, the overall incubation times are 5, 10, 20 , and $30 \mathrm{~min}$, respectively.

In experiments performed with a preincubation, the template was first incubated with a subset of general transcription factors for $30 \mathrm{~min}$ in the presence or absence of GAL4-AH. The remaining factors were then added for 3,8 , or $28 \mathrm{~min}$. GAL4-AH was also added this time if it was lacking in the initial incubation. Therefore, all reactions contained a complete set of factors. Subsequently, dATP and $\alpha$-amanitin were added for $2 \mathrm{~min}$ before $\mathrm{KMnO}_{4}$ probing. Thus, the overall times for the second incubation were 5,10 , or $30 \mathrm{~min}$. To get the 2 -min time point for the second incubation, dATP and $\alpha$-amanitin were premixed with the remaining factors. The whole mixture was then added to that from the preincubation for an additional 2 min before $\mathrm{KMnO}_{4}$ probing.

The kinetic experiments were performed using two different preparations of general factors. Each experiment was done at least twice using each preparation. Only the data from the more active preparation of factors are shown. The data from the less active preparation are consistent with our conclusions. The autoradiographs were scanned with a Bio-Rad densitometer. The intensity of the DNA bands from -3 to +3 was divided by the intensity of the +17 band to yield the relative level of open complex formation shown in Figure 8. The error for the lower ratios was negligible, whereas for the higher ratios, the values varied by about $25 \%$ for two different experiments. Figure 8 shows a representative data set.

\section{Acknowledgments}

This work was supported by NSF grant DMB 88-19713 and NIH grants GM35754 and GM46424. We thank S. Smale, A. Berk, and $\mathrm{A}$. Courey for comments on the manuscript.

The publication costs of this article were defrayed in part by payment of page charges. This article must therefore be hereby marked "advertisement" in accordance with 18 USC section 1734 solely to indicate this fact.

\section{References}

Baker, C.C. and E.B. Ziff. 1981. Promoters and heterogeneous $5^{\prime}$ termini of the messenger RNAs of Adenovinus serotype 2. $I$. Mol. Biol. 149: 189-221.

Berger, S.L., W.D. Cress, A. Cress, S.J. Triezenberg, and L. Guarente. 1990. Selective inhibition of activated but not basal transcription by the acidic activation domain of VP16: Evidence for transcriptional adaptors. Cell 61: 1199-1208.

Borowiec, J.A., L. Zhang, S. Sasse-Dwight, and J.D. Gralla. 1987. DNA supercoiling promotes formation of a bent repression loop in lac DNA. J. Mol. Biol. 196: 101-111.

Bunick, D., R. Zandomeni, S. Ackerman, and R. Weinmann. 1982. Mechanism of RNA polymerase II-specific initiation of transcription in vitro: ATP required and uncapped runoff transcripts. Cell 29: 877-886.

Buratowski, S., S. Hahn, P.A. Sharp, and L. Guarente. 1988. Function of a yeast TATA element-binding protein in a mammalian transcription system. Nature 334: 37.

Buratowski, S., S. Hahn, L. Guarente, and P.A. Sharp. 1989. Five intermediate complexes in transcription initiation by RNA polymerase II. Cell 56: 549-561.

Buratowski, S., M. Sopta, I. Greenblatt and P.A. Sharp. 1991. RNA polymerase II-associated proteins are required for a DNA conformation change in the transcription initiation complex. Proc. Natl. Acad. Sci. 88: 7509-7513.

Carey, M. 1991. Mechanistic advances in eukaryotic gene activation. Curr. Opin. Cell. Biol. 3: 452-460.

Carey, M., I. Leatherwood, and M. Ptashne. 1990a. A potent GAL4 derivative activates transcription at a distance in vitro. Science 247: 710-712.

Carey, M., Y.-S. Lin, M.R. Green, and M. Ptashne. 1990b. A mechanism for synergistic activation of a mammalian gene by GAL4 derivatives. Nature 345: 361-364.

Comai, L., N. Tanese, and R. Tjian. 1992. The TATA-binding protein and associated factors are integral components of the RNA polymerase I transcription factor, SL1. Cell 68: 965976.

Cormack, B.P. and K. Struhl. 1992. The TATA-binding protein is required for transcription by all three nuclear RNA polymerases in yeast cells. Cell 69: 685-696.

Croston, G.E., L.A. Kerrigan, L.M. Lira, D.R. Marshak, and J.T. Kadonaga. 1991. Sequence-specific antirepression of histone $\mathrm{Hl}$-mediated inhibition of basal RNA polymerase II transcription. Science 251: 643-649.

Cullen, B.R. 1990. The HIV-1 Tat protein: An RNA sequencespecific processivity factor? Cell 63: 655-657.

Dignam, J.D., P.L. Martin, B.S. Shastry, and R.G. Roeder. 1983. Eukaryotic gene transcription with purified components. Methods Enzymol. 101: 582-598.

Dynlacht, B.D., T. Hoey, and R. Tjian. 1991. Isolation of coactivators associated with the TATA-binding protein that me- 
diate transcriptional activation. Cell 55: 563-576.

Fire, A., M. Samuels, and P.A. Sharp. 1984. Interactions between RNA polymerase II, factors and template leading to accurate transcription. J. Biol. Chem. 259: 2509-2516.

Flanagan, P.M., R.J. Kelleher, M.H. Sayre, H. Tschochner, and R.D. Kornberg. 1991. A mediator required for activation of RNA polymerase II transcription in vitro. Nature 350: 436438.

Flores, O., H. Lu, M. Killeen, J. Greenblatt, Z.F. Burton, and D. Reinberg. 1991. The small subunit of transcription factor TFIF recruits RNA polymerase II into the preinitiation complex. Proc. Natl. Acad. Sci. 88: 9999-10003.

Gralla, J.D. 1990. Promoter recognition and mRNA initiation by Escherichia coli $\sigma^{70}$. Methods Enzymol. 185: 37-53.

- 1991. Transcriptional control-Lessons from an E. coli promoter data base. Cell 66: 415-418.

Greenblatt, J. 1991. RNA polymerase-associated transcription factors. Trends Biochem. Sci. 16: 408-411.

Ha, I., W.S. Lane, and D. Reinberg. 1991. Cloning of a human gene encoding the general transcription initiation factor TFIIB. Nature 352: 689-695.

Hawley, D.K. and R.G. Roeder. 1985. Separation and partial characterization of three functional steps in transcription initiation by RNA polymerase II. I. Biol. Chem. 260: 83618372.

Horikoshi, M., M. Carey, H. Kakidani, and R.G. Roeder. 1988a. Mechanism of action of a yeast activator: Direct effect of GAL4 derivatives on mammalian TFIID-promoter interactions. Cell 54: 665-669.

Horikoshi, M., T. Hai, Y.-S. Lin, M. Green, and R.G. Roeder. 1988b. Transcription factor ATF interacts with the TATA factor to facilitate establishment of a preinitiation complex. Cell 54: 1033-1042.

Ingles, C.J., M. Shales, W.D. Cress, S.J. Triezenberg, and J. Greenblatt. 1991. Reduced binding of TFIID to transcriptionally compromised mutants of VP16. Nature 351: 588-590.

Kassavetis, G.A., B.R. Braun, L.H. Nguyen, and E.P. Geiduschek. 1990. S. cerevisiae TFIIIB is the transcription initiation factor proper of RNA polymerase III, while TFIIIA and TFIIIC are assembly factors. Cell 60: 235-245.

Kelleher, R.J., P.M. Flanagan, and R.D. Kornberg. 1990. A novel mediator between activator proteins and the RNA polymerase II transcription apparatus. Cell 61: 1209-1215.

Laybourn, P.J. and M.E. Dahmus. 1990. Phosphorylation of RNA polymerase TFIIA occurs subsequent to interaction with promoter and before the initiation of transcription. $I$. Biol. Chem. 265: 13165-13173.

Laybourn, P.J. and J.T. Kadonaga. 1991. Role of nucleosomal cores and histone $\mathrm{Hl}$ in regulation of transcription by RNA polymerase II. Science 254: 238-245.

Lewin, B. 1990. Commitment and activation at polymerase II promoters: A tail of protein-protein interactions. Cell 61: 1161-1164.

Lin, Y.-S. and M. Green. 1991. Mechanism of action of an acidic transcriptional activator in vitro. Cell 64: 971-981.

Lin, Y.-S., M.F. Carey, M. Ptashne, and M.R. Green. 1988. GAL4 derivatives function alone and synergistically with mammalian activators in vitro. Cell 54: 659-664.

Lin, Y.-S., I. Ha, E. Maldonado, D. Reinberg, and M.R. Green. 1991. Binding of general transcription factor TFIIB to an acidic activating region. Nature 353: 569-571.

Lu, H., O. Flores, R. Weinmann, and D. Reinberg. 1991. The nonphosphorylated form of RNA polymerase II preferentially associates with the preinitiation complex. Proc. Natl. Acad. Sci. 88: 10004-10008.

Meiklejohn, A.L. and J.D. Gralla. 1989. Activation of the Iac promoter and its variants. I. Mol. Biol. 207: 661-673.

Meisterernst, M. and R.G. Roeder. 1991. Family of proteins that interact with TFIID and regulate promoter activity. Cell 67: 557-567.

Meisterernst, M., A.L. Roy, H.M. Lieu, and R.G. Roeder. 1991. Activation of class II gene transcription by regulatory factors is potentiated by a novel activity. Cell 66: 981-993.

Mizutani, M., T. Ohta, H. Watanabe, H. Handa, and S. Hirose. 1991. Negative supercoiling of DNA facilitates an interaction between transcription factor TFIID and the fibroin gene promoter. Proc. Natl. Acad. Sci. 88: 718-722.

Nakajima, N., M. Horikoshi, and R.G. Roeder. 1988. Factors involved in specific transcription by mammalian RNA polymerase II: Purification, gene specificity, and TATA box-promoter interactions of TFIID. Mol. Cell. Biol. 8: 4028-4040.

Ohkuma, Y., H. Sumimoto, A. Hoffmann, S. Shimasaki, M. Horikoshi, and R.G. Roeder. 1991. Structural motifs and potential $\mathrm{T}$ homologies in the large subunit of human general transcription factor TFIIE. Nature 354: 398-401

Peterson, M.G., I. Inostroza, M.E. Maxon, O. Flores, A. Admon, D. Reinberg, and R. Tjian. 1991. Structure and functional properties of human general transcription factor TFIIE. Nature 354: 369-373.

Ptashne, M. and A.F. Gann. 1990. Activators and targets. Nature 346: 329-331

Pugh, B.F. and R. Tjian. 1990. Mechanism of transcriptional activation by SP1: Evidence for coactivators. Cell 61: 11871197.

- 1991. Transcription from a TATA-less promoter requires a multisubunit TFIID complex. Genes \& Dev. 5: 1935-1945.

Reinberg, D. and R.G. Roeder. 1987. Factors involved in specific transcription by mammalian RNA polymerase II. $J$. Biol. Chem. 262: 3310-3321.

Reinberg, D., M. Horikoshi, and R.G. Roeder. 1987. Factors in volved in specific transcripiton in mammalian RNA polymerase II. J. Biol. Chem. 262: 3322-3330.

Roeder, R.G. 1991. The complexes of eukaryotic transcription initiation: Regulation of preinitiation complex assembly. Trends Biochem. Sci. 16: 402-408.

Rougvie, A.E. and J.T. Lis. 1988. The RNA polymerase II molecule at the 5 -end of the uninduced hsp70 genes of $D$. melanogaster is transcriptionally engaged. Cell 54: 795-804.

Sasse-Dwight, S. and J.D. Gralla. 1988. Probing the GlnALG upstream activation mechanism in vivo. Proc. Natl. Acad. Sci. 85: 8934-8938.

1989. $\mathrm{KMnO}_{4}$ as a probe for lac DNA melting and mechanism in vivo. I. Biol. Chem. 264: 8074-8081.

Sawadogo, M. and R.G. Roeder. 1984. Energy requirement for specific transcription initiation by the human RNA polymerase II system. J. Biol. Chem. 259: 5321-5326.

Schultz, M.C., R.H. Reeder, and S. Hahn. 1992. Variants of TATA-binding protein can distinguish subsets of RNA polymerase I, II, and III promoters. Cell 69: 697-702.

Sharp, P.A. 1991. TFIIB or not TFIIB? Nature 351: 16-18.

Sopta, M., Z. Burton, and J. Greenblatt. 1989. Structure and associated DNA-helicase activity of a general transcription initiation factor that binds to RNA polymerase II. Nature 341: 410-414.

Stringer, K.F., C.J. Ingles, and J. Greenblatt. 1990. Direct and selective binding of an acidic transcriptional activation domain to the TATA-box factor TFIID. Nature 345: 783-786.

Sumimoto, H., Y. Ohkuma, T. Yamamoto, M. Horikoshi, and R.G. Roeder. 1990. Factors involved in specific transcription by mammalian RNA polymerase II: Identification of general transcription factor TFIIG. Proc. Natl. Acad. Sci. 87: 9158- 
9162.

Sumimoto, H., Y. Ohkuma, E. Sinn, H. Kato, S. Shimasaki, M. Horikoshi, and R.G. Roeder. 1991. Conserved sequence motifs in the small subunit of human general transcription factor TFIIE. Nature 354: 401-404.

Tanese, N., B.F. Pugh, and R. Tjian. 1991. Coactivators for a proline-rich activator purified from the multisubunit human TFIID complex. Genes \& Dev. 5: 2212-2224.

Taylor, I.C., J.L. Workman, T.J. Schuetz, and R.E. Kingston. 1991. Facilitated binding of GAL4 and heat shock factor to nucleosomal templates: Differential function of DNA-binding domains. Genes \& Dev. 5: 1285-1298.

Timmers, H.T.M. and P. Sharp. 1991. The mammalian TFID protein is present in two functionally distinct complexes. Gene ↔ Dev. 5: 1946-1956.

Van Dyke, M.W., R.G. Roeder, and M. Sawadogo. 1988. Physical analysis of transcription preinitiation complex assembly on a class II gene promoter. Science 241: 1335-1338.

Vos, J.C., M. Sasker, and H.G. Stunnenberg. 1991. Promoter melting by a stage-specific vaccinia virus transcription factor is independent of the presence of RNA polymerase. Cell 65: 105-113.

Wang, W., M. Carey, and J.D. Gralla. 1992. Polymerase II promoter activation: Closed complex formation and ATP. driven start-site opening. Science (in press).

Workman, J.L., I.C.A. Taylor, and R.E. Kingston. 1991. Activation domains of stably bound GAL4 derivatives alleviate repression of promoters by nucleosomes. Cell 64: 533-544.

Zawel, L. and D. Reinberg. 1992. Initiation of transcription by RNA polymerase II: A multi-step process. Prog. Nucl. Acids Res. Mol. Biol. (in press).

Zhang, L. and J.D. Gralla. 1989. In situ nucleoprotein structure at the SV40 major late promoter: Melted and wrapped DNA flank the start site. Genes \& Dev. 3: 1814-1822. 


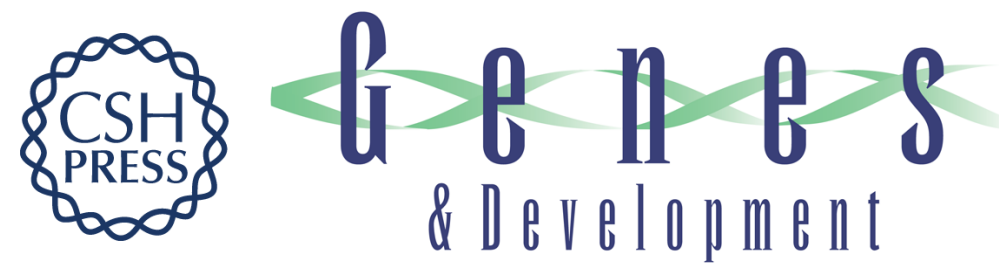

\section{The acidic activator GAL4-AH can stimulate polymerase II transcription by promoting assembly of a closed complex requiring TFIID and TFIIA.}

W Wang, J D Gralla and M Carey

Genes Dev. 1992, 6:

Access the most recent version at doi:10.1101/gad.6.9.1716

References This article cites 66 articles, 22 of which can be accessed free at: http://genesdev.cshlp.org/content/6/9/1716.full.html\#ref-list-1

License

Email Alerting Service

Receive free email alerts when new articles cite this article - sign up in the box at the top right corner of the article or click here.

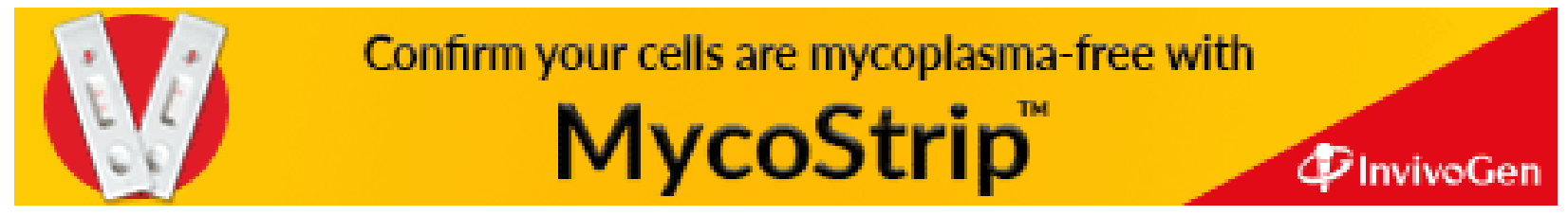

